\title{
Numerical Description of Hot Flow Behaviors at Ti-6Al-2Zr-1Mo-1V Alloy By GA-SVR and Relative Applications
}

\author{
Guo-Zheng Quan ${ }^{a *}$, Zhi-hua Zhang ${ }^{a}$, Yuting Zhou ${ }^{a}$, Tong Wang ${ }^{a}$, Yu-feng Xia ${ }^{a}$ \\ ${ }^{a}$ State Key Laboratory of Mechanical Transmission, School of Material Science and Engineering, \\ Chongqing University, Chongqing, 400044, China
}

Received: April 07, 2016; Revised: July 29, 2016; Accepted: August 20, 2016

\begin{abstract}
Hot compression tests of as-cast Ti-6Al-2Zr-1Mo-1V alloy in a wide temperature range of $1073-1323 \mathrm{~K}$ and strain rate range of $0.01-10 \mathrm{~s}-1$ were conducted by a servo-hydraulic and computer-controlled Gleeble-1500 machine. The hot flow behaviors of Ti-6Al-2Zr-1Mo-1V alloy show highly non-linear relationships with strain, strain rate and temperature. In order to accurately and effectively characterize the complex flow behaviors, support vector regression (SVR) which is a machine learning method was combined with Genetic Algorithm (GA) to characterize the flow behaviors, namely, the GA-SVR. The study abilities, generation abilities, and modeling efficiencies of the improved Arrhenius-type constitutive model, ANN, and GA-SVR for flow behaviors of as-cast Ti-6Al-2Zr-1Mo-1V alloy were detailedly compared. Comparison results show that the study ability of the GA-SVR is as strong as the ANN. The generation abilities and modeling efficiencies of these models were shown as follows in ascending order: the improved Arrhenius-type constitutive model $<$ ANN $<$ GA-SVR. Based on the established GA-SVR, the continuously three-dimensional relationships among flow stress, temperature, strain, and strain rate were constructed, which improve the simulation accuracy and related research fields where stress-strain data play important roles.
\end{abstract}

Keywords: Titanium alloy; Flow stress; Constitutive model; Support vector regression; Genetic Algorithm

\section{Introduction}

Ti-6Al-2Zr-1Mo-1V alloy, a typical near- $\alpha$ titanium alloy, has the advantages of high temperature strength, excellent creep resistance, and good weldability etc., so it was widely utilized for key structural parts in aerospace industry ${ }^{1}$. The existing literatures indicate that there are close relationships among flow stress, strain, strain rate and temperature. It is well known that stress-strain data play important roles in many fields, for examples, reverse analysis from stress-strain data to speculate $\mathrm{WH}$ and $\mathrm{DRV}^{2}$, improving processing maps ${ }^{3}$, and characterizing dynamic recrystallization evolution ${ }^{4}$, etc. An accurate model of flow behaviors is critical to improve material characterization and numerical simulation precision etc. ${ }^{5}$. It is important to establish a model to accurately construct and further predict the highly non-linear flow behaviors. At present, there exist four typical materials constitutive models in modeling hot flow behaviors of metals, namely, empirical/ semiempirical model, analytical model, phenomenological model, and intelligence algorithm ${ }^{6-9}$.

The physical-based analytical model needs explicit and thorough investigation of microscopic deformation

* e-mail: quangz3000@sina.com mechanisms such as the mobile dislocation density, grain coarsening, DRV, and DRX etc. ${ }^{10}$. The physical-based analytical model should deeply understand many microscopic deformation mechanisms and further establish mathematic model for them, otherwise, the physical-based analytical model cannot accurately characterize the highly non-linear flow behaviors ${ }^{11,12}$. Besides, the analytical models require a large amount of precise experiment data to mathematically model complicated microscopic deformation mechanisms ${ }^{13,14}$. Thereby, the analytical models have not been extensively used in characterizing intricate hot flow behaviors.

The phenomenological models do not need to deeply consider complicated microscopic deformation mechanisms, and they only need to calculate requisite material constants and construct multivariate nonlinear regression models according to limited experimental data. Recently, the Arrhenius-type equation and their revised forms of phenomenological models were utilized to model the hot flow behaviors of many materials, such as Ti-6Al- $4 \mathrm{~V}^{15}, \mathrm{Ti}^{16} 0^{16}$, and pure titanium ${ }^{17}$, etc. Lin et al. ${ }^{18,19}$ and Quan et al. ${ }^{20}$ improved the initial Arrhenius-type equations by incorporating strain and some material parameters (such as structure factor $A$ and activation energy of deformation $Q$ ) to obtain more accurate Arrhenius-type equation. Other phenomenological constitutive models involve the typical Johnson-Cook (JC) 
model and Khan-Huang-Liang (KHL) model etc., however, they exhibit large accuracy deviations at different strain rates and temperatures ${ }^{21-24}$. The phenomenological constitutive models cannot accurately track the highly non-linear hot flow behaviors at different strain rates and temperatures, and lack physical models of microscopic deformation mechanisms. And the phenomenological models and empirical models are mathematically fitted based on limited experimental data, showing lower prediction accuracies under unknown deformation conditions ${ }^{23,25}$.

Lately, the artificial neural network (ANN) of intelligence algorithm which imitates biological neural systems was applied in modelling the flow behaviors ${ }^{26}$. .Zhu et al. and Peng et al. respectively constructed ANN models for the flow behaviors of as-cast TC21 titanium alloy ${ }^{27}$ and as-cast Ti60 titanium alloy ${ }^{16}$ during hot deformation, and the correlation coefficients $(R)$ in their work are about 0.992 . The ANN can achieve a high-accuracy level, however, it needs to try a lot of network topologies and training parameters to obtain a higher accuracy, which will consume much time and energy. In addition, ANN is instable. For a certain dataset, the same network topology and training parameters of an ANN will obtain fluctuant accuracies in different attempts, which reduce modelling efficiency. Worse still, ANN is easy to fall into local extreme value and cannot obtain globally optimal solution.

Support vector regression (SVR), as a machine learning method based on statistical learning theory and structural risk minimization principle, is mainly utilized in regression analysis area. SVR has stronger generalization ability and complete theoretical basis. Compared with ANN, SVR can avoid falling into local extreme value and obtain globally optimal solution. A SVR with same training parameters will maintain accuracy at a stable level in different attempts. The computational process of SVR is robust, which guarantees robustness of the prediction model and improves modelling efficiency. SVR does not need to try a lot of network topologies and parameters to achieve a high accuracy level. In this study, SVR was utilized to characterize the hot flow behaviors of Ti-6Al-2Zr-1Mo-1V alloy on account of its excellent advantages. The complexity, learning ability, and generalization ability of SVR depend on the three parameters $(C, \gamma$, and $\zeta$ ), especially the mutual influence among the three parameters. SVR needs to adjust the three parameters (penalty factor $C$, the kernel parameter $\gamma$, and insensitive loss function $\zeta$ ) to obtain an accurate and efficient prediction model. In parameters selection of SVR, optimizing each parameter is unreasonable and time-consuming. The effect of the combination of the three parameters $(C, \gamma$, and $\zeta$ ) on the complexity, learning ability, and generalization of SVR should be synthetically considered. It is inefficient to manually adjust the three parameters one by one to establish an accurate SVR in characterizing the hot flow behaviors for Ti-6Al-2Zr-1Mo-1V alloy. Therefore, it is very important to find a stable and efficient method to realize the optimal selection of the three parameters in SVR. A SVR with the suitable parameters $(C, \gamma$, and $\zeta)$ will accurately learn the stress-strain curves and appropriately ignore some singular points of stress-strain data to accord with the overall trend of the stress-strain curves.

Lou et al. established a SVR combined with particle swarm optimization (PSO) to predict flow stress of AZ80 magnesium alloy where PSO was used to select the parameters $C, \gamma$, and $\zeta$, and the result shows that the model is more accurate than ANN and constitutive equation, besides, the sample dependence of the SVR is lower ${ }^{28}$. Based on SVR, Raghuram Karthik Desu et al. established a prediction model of flow stress for Austenitic Stainless Steel 304, and they found that SVR is more accurate, reliable and efficient than the mathematical regression models such as Johnson-Cook (JC) model, modified-Arrhenius model, modified ZerriliArmstrong (ZA) model, and intelligence algorithm ANN model $^{29}$. The best $R$-value of Raghuram Karthik Desu et al. is 0.9989 at a high accuracy level, however, they just tried a few parameters combinations of the three parameters $(C, \gamma$, and $\zeta)$, and there is still room for improvement in accuracy and efficiency respects ${ }^{29}$. (The evaluation index correlation coefficient $(R)$ was utilized to estimate the degree of correlation between the experimental flow stresses and predicted flow stresses.)

Genetic Algorithm (GA), as a bionic algorithm in solving complex global optimization problem, was enlightened by the Darwin's natural selection theory and the genetic variation theory. The GA has widely used in self-optimizing parameters in various fields on account of the advantages of strong robustness, high efficiency, and parallel processing. In order to utilize the advantages of GA, a SVR model of the hot flow behaviors of Ti-6Al-2Zr-1Mo-1V alloy combined with GA was established where GA was used to efficiently search the optimal parameters combination of the three parameters $(C, \gamma$, and $\zeta$ ), namely, the GA-SVR. The GA-SVR only needs representative training samples from the research, and then self-adaptively and dynamically adjust the three parameters $(C, \gamma$, and $\zeta$ ) to obtain the most accurate SVR. In this work, the comparisons of study abilities, generation abilities and modelling efficiencies among the improved Arrhenius-type constitutive model, ANN, and GA-SVR were investigated. A standard statistical parameter, average absolute relative error $(A A R E)$, was applied to estimate the prediction performance of these models. Comparisons of the results show that the ANN and GA-SVR can sufficiently and accurately learn the hot flow behaviors. In the comparisons of generation abilities, the GA-SVR has larger $R$-value and lower $A A R E$-value, which indicate that the GA-SVR can accurately predict the highly non-linear flow behaviors. The generation abilities of these three models were shown as follows in ascending order: the improved Arrhenius-type constitutive model $<$ ANN $<$ GA-SVR. The GA-SVR self- 
adaptively and dynamically adjusts the three parameters $(C, \gamma$, and $\zeta$ ) to obtain the most accurate SVR, which greatly improves the computational efficiency than ANN. The modeling efficiencies of these models were shown as follows in ascending order: the improved Arrhenius-type constitutive model $<$ ANN $<$ GA-SVR.

An accurate and continuous database of stress data will improve the related research fields where stress-strain data play important roles. In the past, Sun et al. and Zhu et al. just predicted unknown stress data at a certain strain and strain rate ${ }^{27,30-33}$. In this work, a continuously three-dimensional (3D) prediction map of stress data was constructed to represent stress data at any temperature, strain and strain rate. The continuous full-scale database of stress data can improve the related research fields where stress-strain data play important roles.

\section{Acquisition of experimental stress-strain data}

The chemical compositions (wt. \%) of the adopted Ti-6Al-2Zr-1Mo-1V alloy are as follows: Al-6.30, Zr-1.9, V-1.68, Mo-1.32, Fe-0.04, C-0.01, Ni-0.01, Ti (balance). The following experimental procedures were according to ASTM Standard: E209-00. The homogenized metal bar of Ti-6Al-2Zr-1Mo-1V alloy was machined by wireelectrode cutting to several specimens with a height of 12 $\mathrm{mm}$ and diameter of $10 \mathrm{~mm}$. Figure 1 shows the optical microstructure of the as-received Ti-6Al-2Zr-1Mo-1V alloy with single $\alpha$-phase, little $\beta$-phase and negligible impurities. These specimens were compressed on a servo-hydraulic and computer-controlled Gleeble-1500 machine. The graphite lubricants were used to coat the contact surfaces of the anvils and test samples to reduce the friction and prevent bonding. The test samples were heated at a rate of $5 \mathrm{~K} / \mathrm{s}$ and held at a certain temperature for $3 \mathrm{~min}$ to assure a uniform temperature and reduce material anisotropism. The 24 test samples were compressed with a height reduction $60 \%$ (true strain 0.9163 ) at the strain rates of $0.01,0.1,1$, and $10 \mathrm{~s}^{-1}$, and the temperatures of $1073 \mathrm{~K}, 1123 \mathrm{~K}, 1173 \mathrm{~K}, 1223 \mathrm{~K}$, $1273 \mathrm{~K}$ and $1323 \mathrm{~K}$, and then these compressed test samples were rapidly quenched into water to retain the microstructures acquired at high temperatures. During these compressions, a personal computer which is equipped with an automatic data acquisition system was utilized to continuously record the nominal stress and nominal strain, and then the data were converted into true strain and true stress based on the following formulae: $\varepsilon_{\mathrm{T}}=\ln \left(1-\varepsilon_{\mathrm{N}}\right)$ and $\sigma_{\mathrm{T}}=\sigma_{\mathrm{N}}\left(1-\varepsilon_{\mathrm{N}}\right)$, where $\varepsilon_{\mathrm{N}}$ is the nominal strain; $\varepsilon_{\mathrm{T}}$ is the true strain; $\sigma_{\mathrm{T}}$ is the true stress; and $\sigma_{\mathrm{N}}$ is the nominal stress.

Figure 2 shows the experimental true compressive stress-strain curves of Ti-6Al-2Zr-1Mo-1V alloy at different strain rates and temperatures. It can be summarized that the flow stress level increases with the increase of strain

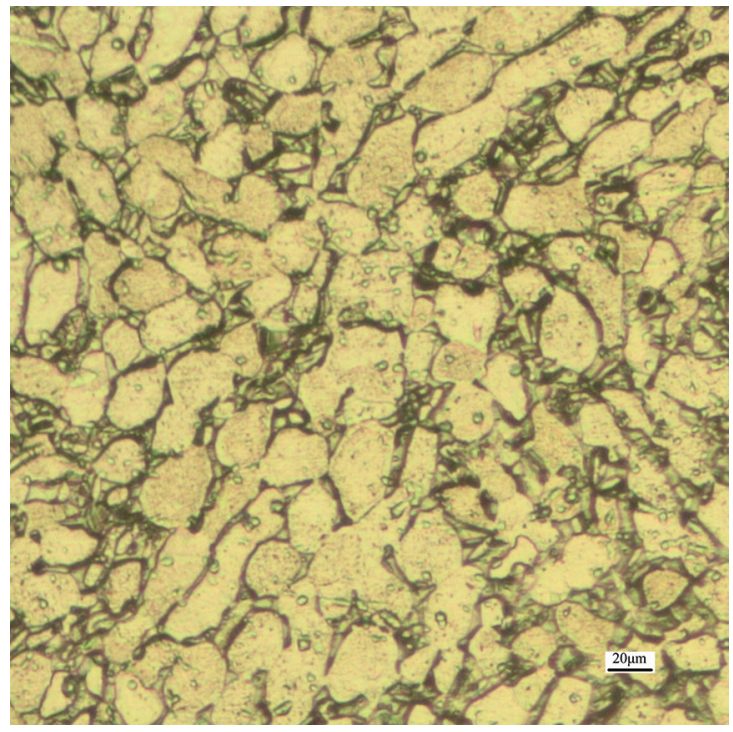

Figure 1: Optical photographs of the as-received Ti-6Al-2Zr1Mo-1V alloy.

rate for a certain temperature, and the flow stress level decreases signally with the increase of temperature for a certain strain rate. The true stress evolution with strain can be roughly classified into three stages. At the first deformation stage, the stress rapidly increases to a critical value with the increase of strain where work hardening (WH) dominates this deformation stage, in the meantime, the stored energy in grain boundaries increases rapidly to the activation energy of dynamic recrystallization (DRX). The recrystallization nucleation will occur when a critical activation energy is achieved. At the second stage, DRX and dynamic recovery (DRV) occur and increase, and the increasing rate of flow stress decelerates until a maximum stress where the thermal softening on account of DRX and DRV are balanced with $\mathrm{WH}^{34,35}$. The flow stress evolution exhibits two types at the third stage: the flow stress approximately keeps at a stable level which indicates a new dynamic balance between hardening due to the dislocation accumulation and softening due to dominant DRV and slight DRX (1173-1323 K \& 0.01-0.1 s ${ }^{-1}$, and 1223-1323 $\left.\mathrm{K} \& 1-10 \mathrm{~s}^{-1}\right)^{34,35}$; the flow stress continuously decreases with distinct softening due to dominant DRX and slight DRV(1073-1123 K \& 0.01-0.1 s $\mathrm{s}^{-1}$, and 1073-1173 K \& $\left.1-10 \mathrm{~s}^{-1}\right)^{34,35}$. The existing literatures indicate that there are close relationships among flow stress, strain, strain rate and temperature. It is well known that stress-strain data play important roles in many fields, for examples, reverse analysis from stress-strain data to speculate work hardening $(\mathrm{WH})$ and dynamic recovery $(\mathrm{DRV})^{2}$, improving processing maps ${ }^{3}$, characterizing dynamic recrystallization evolution $^{4}$, etc. Therefore, it is important to establish a model to accurately and efficiently characterize the highly non-linear flow behaviors. 


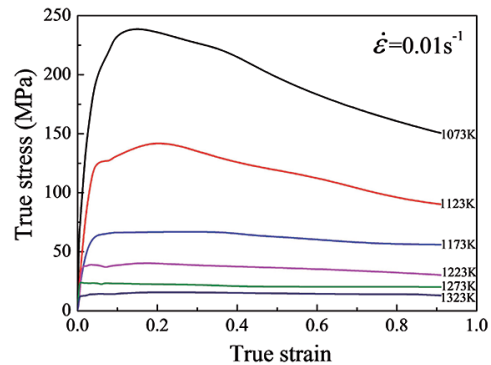

(a)

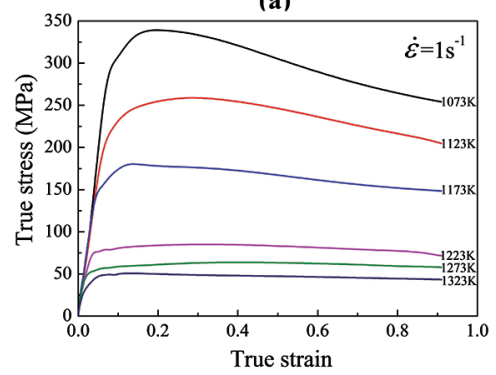

(c)

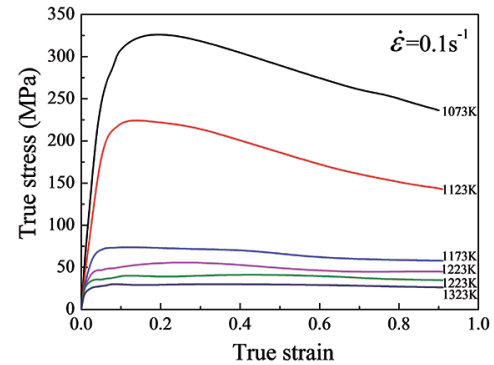

(b)

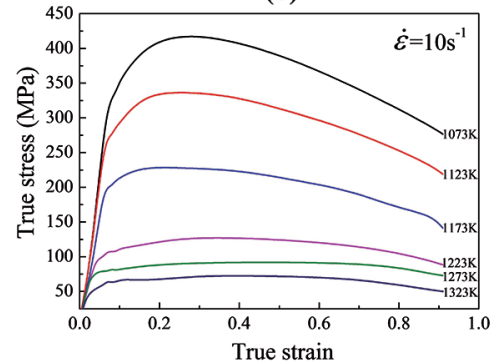

(d)

Figure 2: True stress-strain curves for Ti-6Al-2Zr-1Mo-1V alloy under different strain rates and temperatures.

\section{Development of support vector regression (SVR) for the flow behaviors of as-cast Ti- 6Al-2Zr-1Mo-1V}

In this investigation, support vector regression (SVR) was used to establish the flow behaviors model of Ti-6Al$2 \mathrm{Zr}-1 \mathrm{Mo}-1 \mathrm{~V}$ alloy on account of the excellent regression analysis ability, robustness, and high efficiency of SVR.

\subsection{The basic principles of SVR}

Support vector machine (SVM) is a machine learning method based on statistical learning theory and structural risk minimization principle. With the help of kernel function in SVM, the linearly inseparable low-dimensional data are mapped into linearly separable multidimensional data which can be used for classification and regression analysis. Thereby, SVM is mainly utilized in classification and regression analysis area, which is classified into support vector classification (SVC) and support vector regression (SVR).

The main advantages of SVR are as follows. Firstly, with the help of kernel function, SVR can avoid the curse of dimensionality. Secondly, in SVR, the linearly inseparable low-dimensional data are mapped into linearly separable high-dimensional data, and then SVR constructs the linear discriminant function in high dimension space to realize the nonlinear discrimination in original space. Thirdly, compared with artificial neural network (ANN), the globally optimal solution can be obtained by using SVR. The computational process of SVR is robust and will avoid falling into local extreme value. SVR has strong generalization ability and complete theoretical basis, and does not need to try a lot of network topologies to obtain a highly accurate model.

For a nonlinear problem, the linearly inseparable low-dimensional data are mapped into linearly separable multidimensional data by kernel function, and this mapping can be briefly expressed as Eq. (1):

$$
\begin{aligned}
& \chi \longrightarrow \Phi(\chi)=\left(e_{1} \Phi_{1}(\chi), e_{2} \Phi_{2}(\chi), \ldots, e_{n} \Phi_{n}(\chi)\right) \\
& e_{n} \in R, \Phi_{n} \in R
\end{aligned}
$$

where $x$ is input variable; $\Phi(x)$ is mapping function; $e_{1}, e_{2}, \ldots, e_{n}$ are constants. For example, a two-dimensional data are mapped in a six dimensional space by a second-order polynomial, as expressed by Eq. (2).

$$
\left(\chi_{1}, \chi_{2}\right) \longrightarrow \Phi\left(\chi_{1}, \chi_{2}\right)=\left(1, \chi_{1}, \chi_{2}, \chi_{1}^{2}, \chi_{1} \chi_{2}, \chi_{2}^{2}\right)
$$

In SVR, the mapping is realized by kernel function $k\left(x_{i}, x_{j}\right)=\Phi\left(x_{i}\right) \cdot \Phi\left(x_{j}\right)$. The original data can be mapped in infinite dimensional feature space by the radial basis function $(\mathrm{RBF})$, so the limited data in this feature space can be linearly separated. And a SVR equipped with the RBF can achieve a higher regression precision. Therefore, the RBF expressed as Eq. (3) was used in this investigation.

$$
k\left(\chi_{i}, \chi\right)=\exp \left(-\gamma\left\|\chi_{i}-\chi\right\|^{2}\right), \gamma=\frac{1}{2 \tau^{2}}
$$

where $\gamma$ and $\tau^{2}$ are variable parameters of the RBF. An appropriate parameter $\tau^{2}$ will avoid under-fitting and over-fitting of data in SVR.

The $y=f(x)$ in SVR can be expressed as Eq. (4): 


$$
f(\chi)=\omega \cdot \chi+b
$$

where $\boldsymbol{\omega}$ is a multidimensional column vector; $b$ is a bias term. Assuming that the original data are $\left(x_{1}, y_{1}\right),\left(x_{2}, y_{2}\right),\left(x_{3}, y_{3}\right), \ldots,\left(x_{i}, y_{i}\right), \ldots,\left(x_{l}, y_{l}\right), x_{i}, y_{i} \in R$ . It is assumed that a function $f(x)$ is able to estimate all data, and the optimal function can be expressed as:

$$
\min \frac{1}{2}\|w\|^{2}+C \sum_{i=1}^{l}\left(\xi_{i}+\xi_{i}^{*}\right)
$$

$$
\begin{aligned}
& \text { s.t. } \\
& \left\{\begin{array}{l}
y_{i}-\omega \cdot x_{i}-b \leq \zeta+\xi_{i} \\
\omega \cdot x_{i}+b-y_{i} \leq \zeta+\xi_{i}^{*} \\
\xi_{i}, \xi_{i}^{*} \geq 0
\end{array}\right.
\end{aligned}
$$

where $\xi_{i}$ and $\xi_{i}^{*}$ are slack variables which can improve regression precision; $\boldsymbol{\omega}$ is a multidimensional column vector; $C$ is the penalty factor; $\zeta$ is an insensitive loss parameter which greatly impacts regression precision of SVR. In this work, the input variables $x$ of SVR contain strain $(\varepsilon)$, strain rate $(\varepsilon)$ and temperature $(T)$, and the target output $f(x)$ is flow stress $(\sigma)$ of Ti-6Al-2Zr-1Mo-1V alloy.

The regression function of this optimal hyperplane in SVR can be expressed as Eq. (7):

$$
f(\chi)=\sum_{i=1}^{l}\left(\alpha_{i}-\alpha_{i}^{*}\right) k\left(\chi_{i}, \chi\right)+b
$$

where $\alpha_{i}$ is Lagrange multiplier; $b$ is a bias term; $k\left(x_{i}, x\right)$ is a kernel function.

\subsection{The influence of parameters selection on the performance of SVR}

In SVR, the learning performance and prediction performance can be improved by proper parameters settings, and such parameters are penalty factor $C$ (expressed by Eq. (5)), the kernel parameter $\gamma$ (expressed by Eq. (3)), and insensitive loss function $\zeta$ (expressed by Eq. (6)).

(1) Penalty factor $C$

The complexity and robustness of SVR are influenced by the penalty factor $C$-value. A larger $C$-value in SVR indicates that all of data samples are important and each sample in optimal hyperplane should be correctly classified, which will cause the model to be complex and over-fitting. While a smaller $C$-value in SVR indicates that some singular points can be ignored. However, if the penalty factor $C$-value is too small, the SVR will show the phenomenon of under-fitting.

(2) The parameter $\gamma$ of basis kernel function (RBF).

The RBF expressed as Eq. (3) was used in this investigation. The parameter $\tau^{2}$ determines data distribution in multidimensional feature space and even impacts the generalization ability and learning ability of SVR. There exist severe over-fitting in the following cases: (a) penalty factor $C$-value is set as a certain value and $\tau^{2} \rightarrow 0$; (b) $\tau^{2}$ is set as a certain value and $C \rightarrow \infty^{36}$. And there exist severe under-fitting in the following cases: (a) $C$ is set as a certain value and $\tau^{2} \rightarrow \infty$; (b) $C$ is set as a smaller value and $\tau^{2} \rightarrow 0$ ; (c) $\tau^{2}$ is set as a certain value and $C \rightarrow 036$.

(3) The insensitive loss function $\zeta$.

In SVR, the $\zeta$-value influences the number of support vector and further impacts the regression precision of the model.

It can be summarized that the complexity, learning ability, and generalization ability of SVR depend on the three parameters $C, \gamma$, and $\zeta$, especially the mutual influence among the three parameters. In parameters selection of SVR, optimizing each parameter is unreasonable and time-consuming. The effect of the combination of the three parameters $(C, \gamma$, and $\zeta$ ) on the complexity, learning ability, and generalization of SVR should be synthetically considered. It is inefficient to manually adjust the three parameters one by one to establish an accurate SVR in characterizing the hot flow behaviors for Ti-6Al-2Zr-1Mo-1V alloy. Therefore, it is very important to find a precise, stable and efficient method to realize the optimal selection of the three parameters in SVR. A SVR with suitable parameters $C, \gamma$, and $\zeta$ will accurately learn the stress-strain curves and appropriately ignore some singular points of stress-strain data to accord with the overall trend of the stress-strain curves.

\subsection{The stress prediction model based on SVR and Genetic Algorithm (GA)}

In this section, Genetic Algorithm (GA) was combined with SVR to establish the flow stress prediction model of the hot flow behaviors of Ti-6Al-2Zr-1Mo-1V alloy where GA was used to efficiently search the optimal parameters combination of the three parameters $(C, \gamma$, and $\zeta$ ), and the model was called as GA-SVR in this work.

\subsubsection{The basic principles of $G A$}

Genetic Algorithm is a bionic algorithm in solving complex global optimization problem, which was enlightened by the Darwin's natural selection theory and the genetic variation theory $^{37}$. GA, as a global optimization algorithm, has widely used in various fields on account of the advantages of strong robustness, high efficiency, and parallel processing. GA seeks the optimal solution in solution space by imitating the natural selection process and natural genetic mechanism.

In GA, a population is composed of a certain number of individuals which are encoded by gene encoding. After generation of initial population, optimal approximate solutions are evolved in every generation. The individuals are selected by using fitness function in each generation. According to the fitness value of each individual, the individual which has a 
higher fitness value is inherited to the next generation with greater probability. And the individuals cross and mutate to generate new population which represents new solution. The subsequent generated populations will adapt to environment better than the populations in previous generations. The best individual of last population after decoding is outputted as an approximate optimal solution.

\subsubsection{The establishment of stress prediction model GA-SVR}

In order to utilize the advantages of GA, it was combined with SVR to establish the flow stress prediction model of the hot flow behaviors of Ti-6Al-2Zr-1Mo-1V alloy where GA was used to efficiently search the optimal parameters combination of the three parameters $(C, \gamma$, and $\zeta$ ), namely, the GA-SVR.

In this work, the 24 stress-strain curves were separated into two datasets, namely, the training dataset and independent test dataset, as shown in Table 1. The 864 input-output pairs were selected from the stress-strain curves to train and test the GASVR. The 36 stress points of the testing stress-strain curves at the strain range of 0.1-0.9 with a distance of 0.1 were not utilized for training but for testing the generation ability of the GA-SVR. The 820 stress points of the training stress-strain curves at the strain range of $0.1-0.9$ with a distance of 0.02 were utilized to train the GA-SVR. And the 8 stress points of the testing stress-strain curves at strain of 0.12 and 0.88 were used to train the GA-SVR.

The cross validation method, an effective method in evaluating the accuracy of data mining and machine learning, was utilized in this investigation to evaluate the accuracy of the established GA-SVR. In cross validation method, the original data are divided into $N$ data sets. A separate sample is retained as a validation value and the other $(N-1)$ samples are used to train the GA-SVR. Each sample of $N$ data sets is alternately set as validation data, and the performance of the GA-SVR is evaluated by the average number of the calculated evaluation index in $N$ validation process. In this work, the number $N$ was set as 5 . An evaluation index mean square error (MSE) between training stress data and validation stress data was introduced as Eq. (8).

$$
M S E=\frac{1}{N} \sum_{i=1}^{N}\left[f\left(\chi_{i}\right)-y_{i}\right]^{2}
$$

where $f\left(x_{i}\right)$ are the predicted stress data; $y_{i}$ are the experimental stress data. And $\mathrm{N}$ is the number of stress-strain samples of validation stress dataset.

Besides, other evaluation index correlation coefficient $(R)$ expressed as Eq. (9) was utilized to estimate degree of correlation between the experimental flow stresses and predicted flow stresses ${ }^{38}$. A larger $R$-value demonstrates a well correlation between the two variables, and vice versa.

$$
R=\frac{\sum_{i=1}^{N}\left(E_{i}-\bar{E}\right)\left(P_{i}-\bar{P}\right)}{\sqrt{\sum_{i=1}^{N}\left(E_{i}-\bar{E}\right)^{2} \sum_{i=1}^{N}\left(P_{i}-\bar{P}\right)^{2}}}
$$

where $E$ is the sample of experimental stress-strain data; $P$ is the sample of predicted stress-strain data; $N$ is the number of samples of testing dataset.

The specific flowchart of the GA-SVR was illustrated in Figure 3.

Step 1. Initialize the population of the GA-SVR. The parameters of the $C, \gamma$, and $\zeta$ were encoded to the chromosomes. In this investigation, the binary encoding was adopted to express individuals, because the processes of encoding and decoding operation, crossover operation, and mutation operation in binary encoding are efficient. And the binary encoding is easily analyzed by schema theorem. Here, the population number was set as 20 .

Step 2. The fitness values of the individuals were calculated by fitness function expressed by Eq. (8) in GA.

Step 3. The population was updated by the operators of selection, crossover, and mutation. According to the fitness value of each individual, the individual which has a smaller $M S E$-value was inherited to the next generation with greater probability. Crossover probability $P_{\mathrm{C}}$-value is generally set in the range of 0.6 to 0.9 . A larger $P_{\mathrm{C}}$-value will quickly bring new chromosomes to the population, however, it will increase the risk of premature convergence and the loss of excellent gene structure. While a smaller $P_{\mathrm{C}}$-value will delay genetic evolution process. Here, the $P_{\mathrm{C}}$-value was set as 0.7 . When the searching space of GA adjoins the optimal solution by using the crossover operator, the local random search ability of the mutation operator can be used to accelerate the convergence of the optimal solution, thereby, the mutation probability $P_{\mathrm{m}}$-value should be set as a smaller value. Here, $P_{\mathrm{m}}$-value was set as 0.01 . The cross validation method was used to evaluate the accuracy of GA-SVR.

Table 1: The partition of training dataset and test dataset of stress-strain curves.

\begin{tabular}{lcccc}
\hline \multirow{2}{*}{ Temperature $/ \mathrm{K}$} & \multicolumn{3}{c}{ Strain rate $/ \mathrm{s}^{-1}$} & \\
\\
\hline 1073 & 0.01 & 0.1 & Training & Training \\
1123 & Training & Training & Testing & Training \\
1173 & Training & Training & Training & Training \\
1223 & Testing & Training & Training & Training \\
1273 & Training & Training & Training & Testing \\
1323 & Training & Testing & Training & Training \\
\hline
\end{tabular}




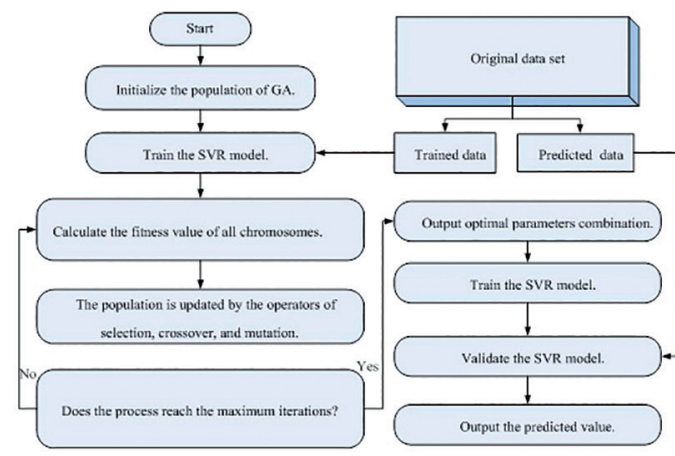

Figure 3: The specific flowchart of the GA-SVR.

Step 4. Stop criterion. If the iteration times achieves the predetermined times, the process of GA-SVR was stopped, and then the optimal parameters were used to train the GASVR. Otherwise, the cyclic process as shown in Figure 3 will constantly proceed. Here, the iteration times was set as 100 .

Figure 4 shows the best fitness value and average fitness value corresponding to iteration times of the well trained GA-SVR. As shown in Figure 4, it can be observed that the convergence speed of the well trained GA-SVR is fast. In the first 10 iteration times, the average fitness value is approaching to the best fitness value state. After the follow-up micro adjustments, the average fitness values eventually achieve the best fitness values in 10 to 20 iteration times. The $C, \gamma$, and $\zeta$ of the best parameters combination $(R=0.999850)$ are $99.61,26.19$, and 0.08 , respectively.

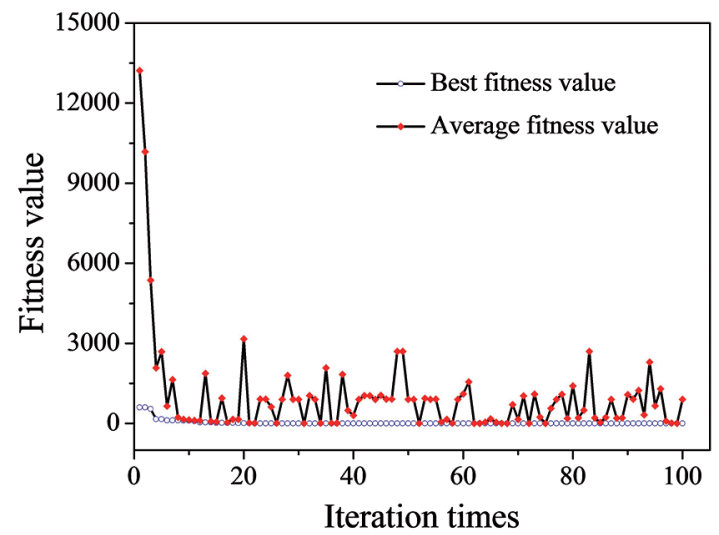

Figure 4: The relationship between the fitness values and the iteration times of the GA-SVR.

\section{Comparisons of the improved Arrhenius- type constitutive model, ANN, and GA-SVR}

In this chapter, the study abilities, generation abilities, and modelling efficiencies of the existing improved Arrheniustype constitutive model, ANN, and GA-SVR for as-cast Ti-6Al-2Zr-1Mo-1V alloy were detailedly compared.

\subsection{The existing improved Arrhenius-type constitutive model and ANN for as-cast Ti- 6Al-2Zr-1Mo-1V alloy}

The original Arrhenius-type constitutive model expressed as Eq. (10) does not consider the influence of strain. Afterwards, Quan et al. calculated the improved Arrheniustype constitutive model for as-cast Ti-6Al-2Zr-1Mo-1V alloy in the reference ${ }^{8}$, which was incorporated with the influence of strain, as expressed by Eq. (11).

$$
\sigma=\frac{1}{\alpha} \ln \left\{\begin{array}{l}
\left(\frac{\dot{\varepsilon} \exp (Q / 8.314 T)}{A}\right)^{\frac{1}{n}}+ \\
{\left[\left(\frac{\dot{\varepsilon} \exp (Q / 8.314 T)}{A}\right)^{\frac{2}{n}}+1\right]^{\frac{1}{2}}}
\end{array}\right.
$$

where $\sigma$ is flow stress (MPa) for a certain strain; $T$ is temperature $(\mathrm{K}) ; Q$ and $A$ are the activation energy $\left(\mathrm{kJ} \cdot \mathrm{mol}^{-1}\right)$ and structure factor of Ti-6Al-2Zr-1Mo-1V alloy, respectively; $\alpha$ and $n$ are the material constants of Ti-6Al2Zr-1Mo-1V alloy.

$$
\sigma=\frac{1}{g(\varepsilon)} \ln \left\{\begin{array}{l}
\left(\frac{\dot{\varepsilon} \exp (j(\varepsilon) / 8.314 T)}{f(\varepsilon)}\right)^{\frac{1}{\mathrm{~h}(\varepsilon)}}+ \\
{\left[\left(\frac{\dot{\varepsilon} \exp (j(\varepsilon) / 8.314 T)}{f(\varepsilon)}\right)^{\frac{2}{h(\varepsilon)}}+1\right]^{\frac{1}{2}}}
\end{array}\right.
$$

where $f(\varepsilon), g(\varepsilon), h(\varepsilon), j(\varepsilon)$ are multinomial functions of strain for $A, \alpha, n$, and $Q$, respectively, as shown in Eq. (12).

$\left\{\begin{array}{l}Q=B_{0}+B_{1} \varepsilon+B_{2} \varepsilon^{2}+B_{3} \varepsilon^{3}+B_{4} \varepsilon^{4}+B_{5} \varepsilon^{5}+B_{6} \varepsilon^{6} \\ n=C_{0}+C_{1} \varepsilon+C_{2} \varepsilon^{2}+C_{3} \varepsilon^{3}+C_{4} \varepsilon^{4}+C_{5} \varepsilon^{5}+C_{6} \varepsilon^{6} \\ \ln A=D_{0}+D_{1} \varepsilon+D_{2} \varepsilon^{2}+D_{3} \varepsilon^{3}+D_{4} \varepsilon^{4}+D_{5} \varepsilon^{5}+D_{6} \varepsilon^{6} \\ \alpha=E_{0}+E_{1} \varepsilon+E_{2} \varepsilon^{2}+E_{3} \varepsilon^{3}+E_{4} \varepsilon^{4}+E_{5} \varepsilon^{5}+E_{6} \varepsilon^{6}\end{array}\right.$

where $B_{0}, B_{1}, \ldots, B_{6}, C_{0}, C_{1}, \ldots, C_{6}, D_{0}, D_{1}, \ldots, D_{6}$, and $E_{0}$, $E_{1}, \ldots, E_{6}$ are the coefficients of the polynomial for $Q, n, \ln$ $A$, and $\alpha$, respectively.

Quan et al. established the ANN for as-cast Ti-6Al-2Zr$1 \mathrm{Mo}-1 \mathrm{~V}$ alloy in the reference ${ }^{8}$.

\subsection{Comparisons of the study abilities of ANN and GA-SVR}

Figure 5 shows the comparisons between the trained flow stresses and testing flow stresses predicted by the GA-SVR at different strain rates and temperatures. As shown in Figure 5, the training predictions accurately track the trained stressstrain curves in a wide temperature range, strain range, and strain rate range. And the testing predictions also track the trends of the untrained stress-strain curves. The correlation 


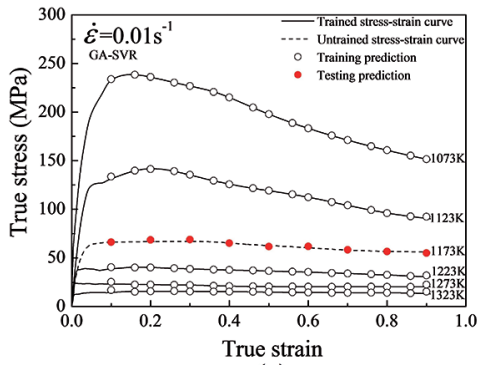

(a)

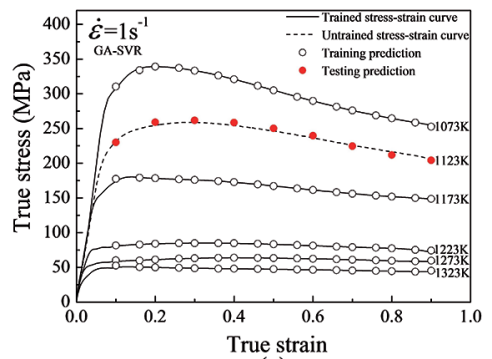

(c)

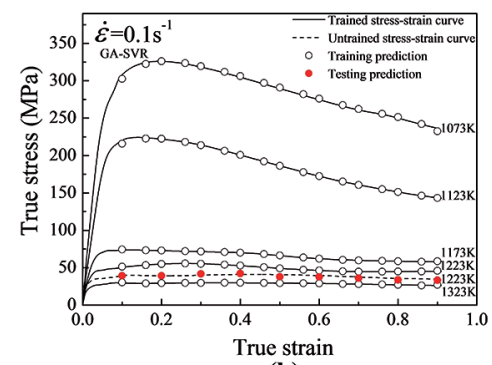

(b)

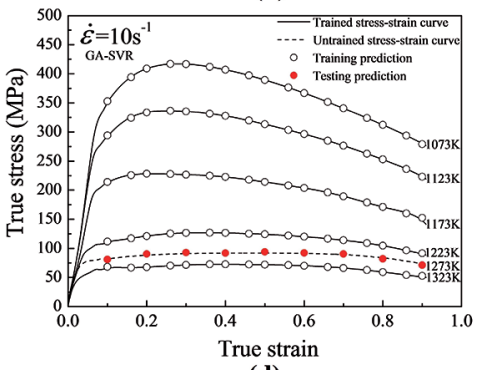

(d)

Figure 5: Comparisons between the trained flow stresses and testing flow stresses predicted by the GA-SVR at different strain rates and temperatures of (a) $0.01 \mathrm{~s}^{-1}, 1073-1123 \mathrm{~K}$, (b) $0.1 \mathrm{~s}^{-1}, 1073-1123 \mathrm{~K}$, (c) $1 \mathrm{~s}^{-1}, 1073-1123 \mathrm{~K}$ and (d) $10 \mathrm{~s}^{-1}, 1073-1123 \mathrm{~K}$.

between the trained| flow stresses and training predictions for the training dataset of the GA-SVR at (a) $0.01 \mathrm{~s}^{-1}$, (b) $0.1 \mathrm{~s}^{-1}$, (c) $1 \mathrm{~s}^{-1}$, and (d) $10 \mathrm{~s}^{-1}$ were calculated and shown in Figure 6. As exhibited in Figure 6, the $R$-values between the training samples and fitted values of the GA-SVR model are larger than 0.9999, and there is no singular point. It can be concluded that the GA-SVR can accurately learn the highly non-linear flow behavior.

In order to further estimate the study abilities of these prediction models, average absolute relative error (AARE) was introduced. $A A R E$ is an average number of the absolute value of relative errors ( $\delta$-values). Relative error $(\delta)$ expressed by Eq. (13) is a typical evaluation index to reflect difference between training data and predicted data. Compared with the $\delta$-value, $A A R E$ expressed by Eq. (14) can better reflect prediction error, because the positive and negative $\delta$-value cannot be offset.

$$
\begin{aligned}
& \delta(\%)=\frac{E_{i}-P_{i}}{E_{i}} \times 100 \% \\
& A A R E=\frac{1}{N} \sum_{i=1}^{N}\left|\frac{E_{i}-P_{i}}{E_{i}}\right|
\end{aligned}
$$

where $E$ is the sample of experimental stress-strain data; $P$ is the sample of predicted stress-strain data; $N$ is the number of samples of testing dataset.

The $R$-values and $A A R E$-values between the training samples and fitted values of the ANN and GA-SVR were listed in Table 2.

As illustrated in Table 2, it can be observed that the $R$-values between the training samples and fitted value of the ANN and GA-SVR model at $0.01 \mathrm{~s}^{-1}, 0.1 \mathrm{~s}^{-1}, 1 \mathrm{~s}^{-1}, 10 \mathrm{~s}^{-1}$ are larger than 0.9999. It can be summarized that both the ANN and GA-SVR model can sufficiently and accurately learn the hot flow behaviors of Ti-6Al-2Zr-1Mo-1V alloy.

\subsection{Comparisons of the generalization abilities of the improved Arrhenius-type constitutive model, ANN, and GA-SVR}

The $\delta$-values between the experimental stress-strain data and testing stress-strain data which were predicted by the improved Arrhenius-type constitutive model, ANN, and GA-SVR were illustrated in Table 3. From Table 3, it can be found that the $\delta$-values acquired from the improved Arrhenius-type constitutive model, ANN, and GA-SVR vary from $-17.31 \%-25.28 \%,-10.85 \%-8.26 \%$, and $-6.78 \%-5.82 \%$, respectively. It is worth noting that a wider fluctuation range of $\delta$-values does not signify poor prediction performance, and the distribution and relative frequency of $\delta$-values should be further analyzed by Gaussian distribution analysis. After Gaussian distribution analysis, the mean number of all relative errors and standard deviation $(w)$ can be obtained. The -value expressed by Eq. (15) is the mean number of all relative errors. The standard deviation (w) expressed by Eq. (16), as an evaluation index to measure discrete degree of individual in the dataset, was introduced to measure the distribution of the relative error $(\delta)$. Here, a small $w$ indicates that most of $\delta$-values are close to -value, and vice versa. And a smaller $\mu$-value indicates that more predicted stress data approach the experimental stress data.

$$
\mu=\frac{1}{N} \sum_{i=1}^{N} \delta_{i}
$$




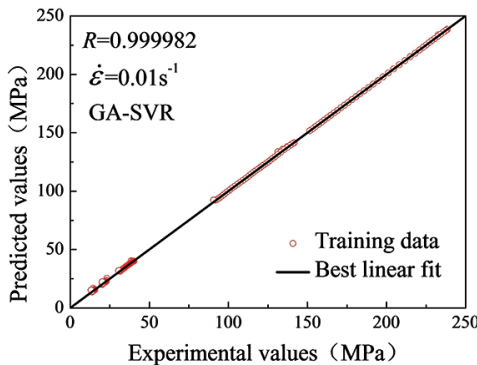

(a)

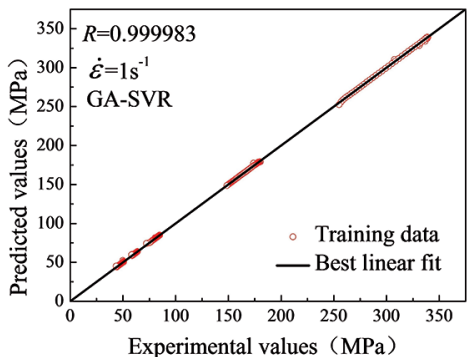

(c)

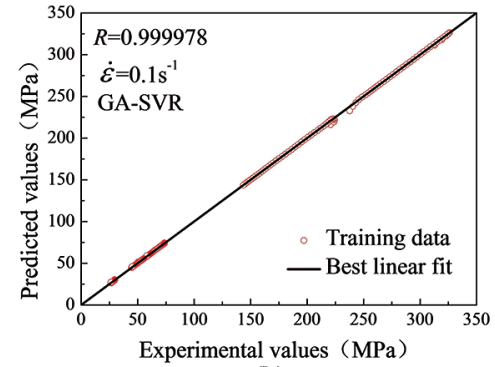

(b)

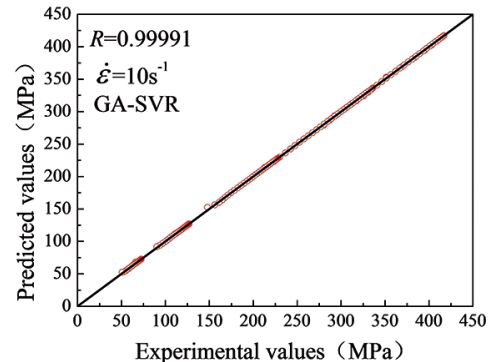

(d)

Figure 6: Correlation between the trained flow stresses and training predictions for the training dataset of the GA-SVR model of (a) 0.01 $\mathrm{s}^{-1}$, (b) $0.1 \mathrm{~s}^{-1}$, (c) $1 \mathrm{~s}^{-1}$, and (d) $10 \mathrm{~s}^{-1}$.

Table 2: $R$-values and $A A R E$-values between the training samples and fitted values of the ANN and GA-SVR at $0.01,0.1,1$, and $10 \mathrm{~s}^{-1}$.

\begin{tabular}{lccc}
\hline Strain rate $/ \mathrm{s}^{-1}$ & \multicolumn{2}{c}{$\begin{array}{c}A A R E \text {-value } \\
\text { GA-SVR }\end{array}$} \\
\hline 0.01 & ANN & GA-SVR & $0.6942 \%$ \\
0.1 & 0.99997 & 0.999982 & $0.2306 \%$ \\
1 & 0.99999 & 0.999978 & $0.2384 \%$ \\
10 & 0.99997 & 0.999983 & $0.1498 \%$ \\
Average & 0.99998 & 0.99991 & $0.3282 \%$ \\
\hline
\end{tabular}

$$
w=\sqrt{\frac{1}{(N-1)} \sum_{i=1}^{N}\left(\delta_{i}-\mu\right)^{2}}
$$

where $\delta$ is the sample of relative error; $\mu$ is the average number of $\delta$-values; $N$ is the number of samples of testing dataset.

Figure $7 \mathrm{a}, \mathrm{b}$, and c show the histogram of $\delta$-values of the improved Arrhenius-type constitutive model, ANN, and GA-SVR, respectively, which show the relative frequency of each $\delta$-level. The $\mu$-value and $w$-value of the improved Arrhenius-type constitutive model, ANN, and GA-SVR are $-2.981 \& 4.202,-1.754$ $\& 2.348$, and $0.585 \& 1.577$, respectively. A smaller $w$-value indicates that most of $\delta$-values are close to the -value, and a smaller $\mu$-value indicates that more predicted stress data approach the experimental stress data. It can be summarized that the generation ability of improved Arrhenius-type constitutive model is the worst, and the generation abilities of the ANN and GA-SVR are at higher levels.

Table 4 exhibits the $R$-values and $A A R E$-values of test datasets of Arrhenius-type constitutive model,
ANN, and GA-SVR. The $A A R E$-values of the improved Arrhenius-type constitutive model, ANN, and GASVR are $7.9703 \%, 4.2163894 .2164 \%$, and $2.1033 \%$, respectively. It can be summarized that the GA-SVR has larger $R$-value and lower $A A R E$-value, which indicate that the GA-SVR can accurately predict the highly nonlinear flow behaviors. The generation abilities of these models were shown as follows in ascending order: the improved Arrhenius-type constitutive model $<$ ANN $<$ GA-SVR. The improved Arrhenius-type constitutive model cannot accurately track the hot flow behaviors, because the mathematical regression method is difficult to describe the complicated non-linear flow behaviors which accompanied with phase transformation, WH, DRV, and DRX in wide temperature and strain rate intervals. Quan et al. established the ANN model for as-cast Ti6Al-2Zr-1Mo-1V alloy with high $R$-value and small $A A R E$-value, however, the input variables just contain deformation temperature $(T)$ and strain $(\varepsilon)^{8}$. The input variables of GA-SVR contain temperature $(\mathrm{T})$, strain $(\varepsilon)$, and strain rate $(\varepsilon)$. 
Table 3: Comparisons between experimental flow stresses and predicted flow stresses for test dataset.

\begin{tabular}{|c|c|c|c|c|c|c|c|c|c|}
\hline \multirow[b]{2}{*}{ Strain rate $/ \mathrm{s}^{-1}$} & \multirow[b]{2}{*}{ Temperature /K } & \multirow[b]{2}{*}{ Strain } & \multirow{2}{*}{$\begin{array}{l}\text { Experimental } \\
\text { stress }(\mathrm{MPa})\end{array}$} & \multicolumn{3}{|c|}{ Predicted stress $(\mathrm{MPa})$} & \multicolumn{3}{|c|}{ Relative error $/ \%$} \\
\hline & & & & Equation & ANN & GA-SVR & Equation & ANN & $\begin{array}{l}\text { GA- } \\
\text { SVR }\end{array}$ \\
\hline \multirow[t]{9}{*}{0.01} & 1173 & 0.1 & 66.16 & 82.88 & 61.88 & 66.22 & 25.28 & -6.46 & 0.09 \\
\hline & & 0.2 & 67.32 & 82.53 & 63.42 & 68.45 & 22.60 & -5.80 & 1.68 \\
\hline & & 0.3 & 66.83 & 80.01 & 62.67 & 68.85 & 19.72 & -6.23 & 3.02 \\
\hline & & 0.4 & 65.32 & 76.23 & 65.51 & 65.13 & 16.70 & 0.29 & -0.29 \\
\hline & & 0.5 & 62.69 & 72.40 & 64.61 & 61.67 & 15.49 & 3.06 & -1.63 \\
\hline & & 0.6 & 60.32 & 68.88 & 61.37 & 61.85 & 14.19 & 1.75 & 2.54 \\
\hline & & 0.7 & 57.88 & 68.22 & 57.86 & 58.36 & 17.88 & -0.02 & 0.83 \\
\hline & & 0.8 & 56.81 & 65.14 & 55.30 & 56.47 & 14.66 & -2.65 & -0.60 \\
\hline & & 0.9 & 56.16 & 62.45 & 50.07 & 55.03 & 11.19 & -10.85 & -2.01 \\
\hline \multirow[t]{9}{*}{0.1} & 1273 & 0.1 & 39.36 & 38.59 & 41.02 & 39.29 & -1.95 & 4.23 & -0.18 \\
\hline & & 0.2 & 38.78 & 39.31 & 41.64 & 39.21 & 1.37 & 7.38 & 1.11 \\
\hline & & 0.3 & 39.55 & 39.13 & 41.25 & 41.85 & -1.05 & 4.31 & 5.82 \\
\hline & & 0.4 & 40.87 & 38.83 & 39.65 & 42.29 & -5.00 & -2.99 & 3.47 \\
\hline & & 0.5 & 40.70 & 38.28 & 37.40 & 37.94 & -5.95 & -8.11 & -6.78 \\
\hline & & 0.6 & 39.34 & 37.36 & 35.45 & 37.71 & -5.04 & -9.88 & -4.14 \\
\hline & & 0.7 & 37.73 & 36.29 & 34.32 & 36.15 & -3.83 & -9.06 & -4.19 \\
\hline & & 0.8 & 35.59 & 34.88 & 33.96 & 33.81 & -2.01 & -4.59 & -5.00 \\
\hline & & 0.9 & 34.84 & 32.51 & 34.15 & 33.42 & -6.69 & -1.97 & -4.08 \\
\hline \multirow[t]{9}{*}{1} & 1123 & 0.1 & 231.49 & 228.16 & 250.62 & 230.08 & -1.44 & 8.26 & -0.61 \\
\hline & & 0.2 & 254.89 & 247.53 & 257.94 & 259.14 & -2.89 & 1.20 & 1.67 \\
\hline & & 0.3 & 258.73 & 246.78 & 254.54 & 261.65 & -4.62 & -1.62 & 1.13 \\
\hline & & 0.4 & 254.81 & 239.10 & 249.13 & 258.42 & -6.17 & -2.23 & 1.42 \\
\hline & & 0.5 & 246.43 & 228.31 & 241.47 & 250.27 & -7.35 & -2.01 & 1.56 \\
\hline & & 0.6 & 236.13 & 216.03 & 232.97 & 239.69 & -8.51 & -1.34 & 1.51 \\
\hline & & 0.7 & 225.99 & 203.78 & 225.08 & 224.72 & -9.83 & -0.40 & -0.56 \\
\hline & & 0.8 & 216.54 & 189.80 & 218.65 & 211.85 & -12.35 & 0.98 & -2.17 \\
\hline & & 0.9 & 206.25 & 170.56 & 213.84 & 204.12 & -17.31 & 3.68 & -1.03 \\
\hline \multirow[t]{9}{*}{10} & 1273 & 0.1 & 81.00 & 84.42 & 83.83 & 80.74 & 4.22 & 3.50 & -0.32 \\
\hline & & 0.2 & 88.18 & 89.33 & 83.78 & 90.54 & 1.29 & -5.00 & 2.68 \\
\hline & & 0.3 & 89.84 & 92.26 & 88.73 & 92.85 & 2.70 & -1.24 & 3.35 \\
\hline & & 0.4 & 91.30 & 93.78 & 90.48 & 91.89 & 2.72 & -0.90 & 0.65 \\
\hline & & 0.5 & 92.27 & 93.18 & 89.98 & 93.83 & 0.98 & -2.48 & 1.69 \\
\hline & & 0.6 & 91.31 & 90.95 & 88.35 & 92.18 & -0.39 & -3.24 & 0.95 \\
\hline & & 0.7 & 89.03 & 88.04 & 84.48 & 90.35 & -1.11 & -5.12 & 1.48 \\
\hline & & 0.8 & 83.78 & 81.74 & 76.77 & 82.06 & -2.43 & -8.36 & -2.05 \\
\hline & & 0.9 & 73.74 & 66.35 & 65.92 & 71.2 & -10.02 & -10.60 & -3.44 \\
\hline
\end{tabular}

\subsection{Comparisons of the modelling efficiencies among the improved Arrhenius-type constitutive model, $A N N$, and GA-SVR}

Table 5 shows the time in modelling an accurate model of the improved Arrhenius-type constitutive model, ANN and GA-SVR. The improved Arrhenius-type constitutive model needs to calculate many material constants and construct many multivariate nonlinear regression models according to limited experimental data. And these material constants and regression models need to be recalculated when new stress data are added. This process is complex and time-consuming. The GA-SVR does not need to establish the complicated mathematical models and the transformation mechanisms of micro structures. 


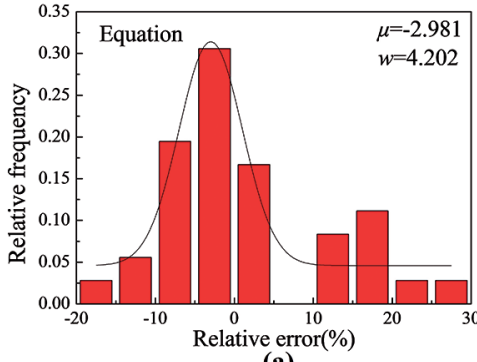

(a)

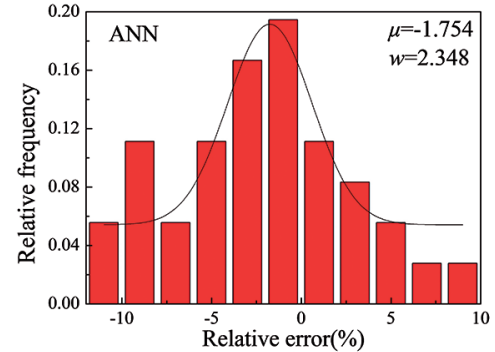

(b)

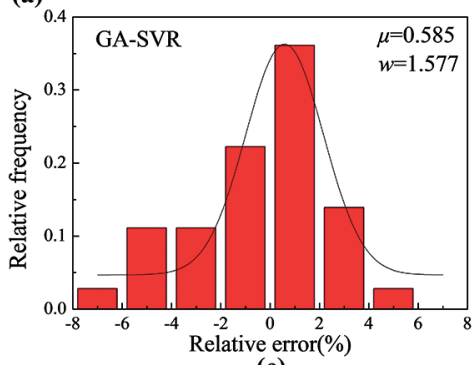

(c)

Figure 7: Distribution of relative errors of test data corresponding to the (a) improved Arrhenius-type constitutive model, (b) ANN, and (c) GA-SVR.

Table 4: $R$-values and $A A R E$-values of test dataset of the improved Arrhenius-type constitutive model, ANN, and GA-SVR.

\begin{tabular}{cccccc}
\hline & \multicolumn{2}{c}{ R-value } & \multicolumn{3}{c}{$A A R E$-value } \\
Arrhenius-type & ANN & GA-SVR & Arrhenius-type & ANN & GA-VR \\
\hline 0.993437 & 0.998309 & 0.999676 & $7.9703 \%$ & $4.2164 \%$ & $2.1033 \%$ \\
\hline
\end{tabular}

Table 5: The time in modelling an accurate model of the Arrhenius-type constitutive model, ANN, and GA-SVR.

\begin{tabular}{lccc}
\hline Model & Arrhenius-type & ANN & GA-SVR \\
\hline The time in modelling an accurate model. & More than $180 \mathrm{~min}$ & More than $60 \mathrm{~min}$ & About 15 min \\
\hline
\end{tabular}

ANN needs to try a lot of network topologies and training parameters to obtain an accurate model, which will consume much time and energy. In addition, ANN is not very stable. To a certain dataset, the same network topology and training parameters of an ANN will obtain fluctuant accuracies in different attempts, which reduces the modelling efficiency. Based on the operators of selection, crossover, and mutation, the GA-SVR can self-adaptively and dynamically adjust the processes of selection, crossover, and mutation to realize the optimal selection of the three parameters, which greatly improves the computational efficiency. Compared with ANN, the globally optimal solution can be obtained by using GA-SVR, and the computational processes of GA-SVR are robust and will avoid falling into local extreme value. GA-SVR does not need to try a lot of network topologies to obtain a highly accurate model. GA-SVR only needs representative training samples from the research, and then automatically adjust the three parameters $C, \gamma$, and $\zeta$ to obtain the most accurate prediction model. Compared with ANN, GA-SVR greatly improves the modeling efficiency. The modeling efficiencies of these models were shown as follows in ascending order: the improved Arrhenius-type constitutive model $<$ ANN $<$ GA-SVR.

\section{Applications of the GA-SVR in material computations}

\subsection{Stress-strain data expansion by the GA-SVR}

In this section, the flow stress data at temperatures of $1098 \mathrm{~K}, 1148 \mathrm{~K}, 1198 \mathrm{~K}, 1248 \mathrm{~K}$, and $1298 \mathrm{~K}$ under strain rates of $0.01 \mathrm{~s}^{-1}, 0.1 \mathrm{~s}^{-1}, 1 \mathrm{~s}^{-1}$ and $10 \mathrm{~s}^{-1}$ were predicted for Ti-6Al-2Zr-1Mo-1V alloy by the GA-SVR, as shown in Figure 8 . The expanded stress-strain data are conducive to the accuracy improvement in the following fields.

\subsection{Accuracy improvement in Finite Element Modeling (FEM)}

If finite element software needs to invoke stress-strain data which are not preliminarily inputted to the software, the software mainly calculates unknown stress-strain data by mathematical interpolation means. However, flow behaviors of materials at different conditions (such as different temperatures and strain rates) are complicated. The interpolation method cannot correctly calculate the stress-strain data, and will obtain inaccurate simulation results. 


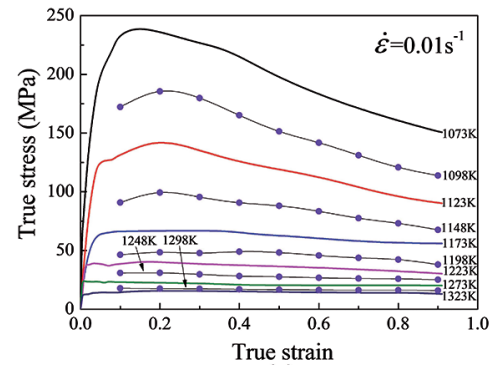

(a)

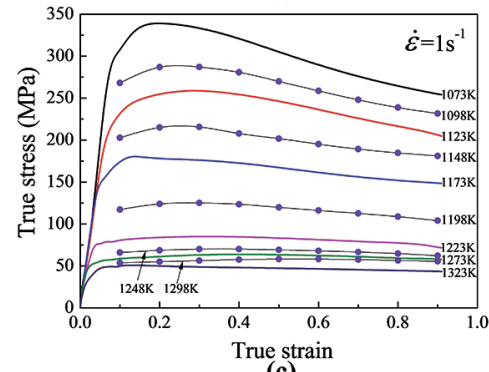

(c)

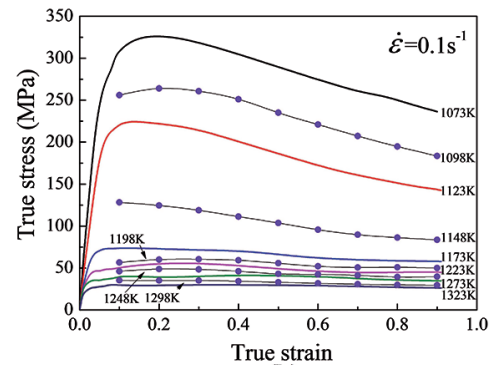

(b)

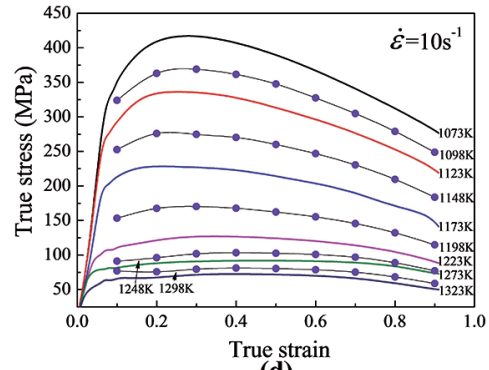

(d)

Figure 8: The true stress-strain curves of Ti-6Al-2Zr-1Mo-1V alloy under different temperatures and different strain rates where the solid curves are experimental data and the fitted curves by points are predicted data.

In this section, the influences of input stress-strain curves on simulation results were analyzed in the isothermal compression experiment by a popular FEM software DEFORM. The simulation parameters were set according to the actual experiment. One half of the specimen was simulated on account of geometric symmetry, so as to decrease the computing time. In the actual experiments, the top and bottom surfaces of specimens were coated with graphite lubricants to decrease friction between the specimen and anvils, therefore, the friction type between the contact surfaces of specimen and dies was set as shear-type in DEFORM. And a shear friction coefficient of 0.3 was set to simulate the actual graphite lubricant condition between the specimens and anvils ${ }^{39}$. In the FEM simulations, the thermal conduction and thermal radiation among compression sample, dies, and ambient were ignored to simulate the experimental isothermal compression test.

Table 6 shows three simulation schemes which were used to analyze the influences of input stress-strain curves on final simulation results. The entire initial conditions were selfsame except for the different input stress-strain curves. The compression tests were simulated at the temperature of $1173 \mathrm{~K}$ and strain rate of $0.01 \mathrm{~s}^{-1}$. The entire experimental stress-strain curves were inputted to the FEM software in Scheme-A, and there is no interpolation interval in scheme-A. The experimental stress-strain curves and the stress-strain curves predicted by the GA-SVR were applied to scheme-B. The experimental stress-strain curves at temperatures of $1073,1123,1223,1273$, and $1323 \mathrm{~K}$ and strain rate of 0.01 $\mathrm{s}^{-1}$ were adopted by scheme-C, so the stress-strain curve at temperature of $1173 \mathrm{~K}$ and strain rate of $0.01 \mathrm{~s}^{-1}$ needs to be interpolated, and the interpolation interval was $100 \mathrm{~K}$.
Figure $9 \mathrm{~b}$ displays the distribution of effective strain of scheme-B, which can be roughly divided into three districts. The distribution of effective strain of scheme-B is similar to scheme-A, and the average strain of scheme-B is 0.953 approaching to scheme-A. Figure 9c displays the distribution of effective strain of scheme-C, which can be similarly divided into three districts. However, there are large differences of effective strain distributions between scheme-C and scheme-A, as well as the maximum effective strain. Besides, the shape of the compression sample of scheme-C is not a typical drum-type on account of the bad interpolation in a wide interpolation interval.

Additionally, as exhibited in Figure 10, the load curves corresponding to strokes of the top dies of these schemes show that the load curves of top dies of scheme-B and scheme-A are very close. The load curves of top dies of scheme-B and scheme-A are close to the experimental loads. However, there are large differences of top die loads between scheme-C and scheme-A. The relative errors of the top die loads between scheme-A and scheme-B are in the range of $-1.3818 \%-2.3872 \%$, whereas this errors between scheme-A and scheme-C are in the range of $0.7158 \%-34.2327 \%$.

It can be concluded that a large span of interpolation or insufficient stress-strain data will lead to inaccurate simulation results. In addition, the flow behaviors under different temperature ranges of a material are highly nonlinear, thereby, calculating stress data by interpolation method in FEM software is inaccurate. There are many fine controls in manufacturing industry, which requires accurate and sufficient material data. The insufficient material data and 
Table 6: The three finite element simulation schemes at the strain rate of $0.01 \mathrm{~s}^{-1}$ and temperature of $1173 \mathrm{~K}$.

\begin{tabular}{lccc}
\hline Temperature (K) & F & Finite element simulation schemes & \\
& Experimental curve & Experimental curve & Experimental curve \\
1073 & Null & Predicted curve by the GA-SVR & Null \\
1123 & Experimental curve & Experimental curve & Experimental curve \\
1148 & Null & Predicted curve by the GA-SVR & Null \\
1173 & Experimental curve & Predicted curve by the GA-SVR & Interpolation of FEM software \\
1198 & Null & Predicted curve by the GA-SVR & Null \\
1223 & Experimental curve & Experimental curve & Experimental curve \\
1248 & Null & Predicted curve by the GA-SVR & Null \\
1273 & Experimental curve & Experimental curve & Experimental curve \\
1298 & Null & Predicted curve by the GA-SVR & Null \\
1323 & Experimental curve & Experimental curve & Experimental curve \\
\hline
\end{tabular}

(a)
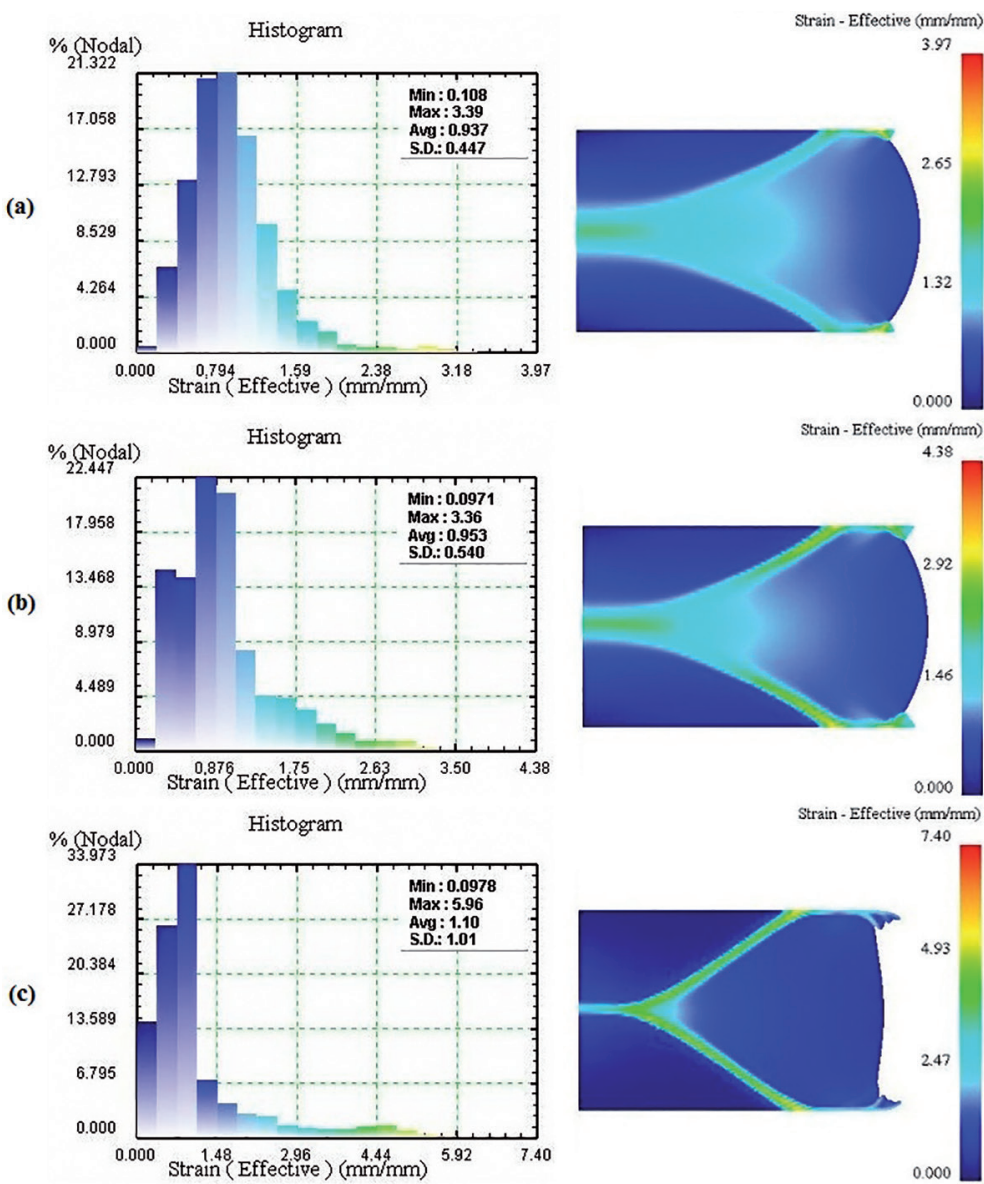

Figure 9: Distributions of effective strain for (a) scheme-A, (b) scheme-B, and (c) scheme-C, at the strain rate of $0.01 \mathrm{~s}^{-1}$, the temperature of $1173 \mathrm{~K}$, and the height reduction of $60 \%$.

inaccurate simulation results will result in huge economic losses. It can be concluded that the GA-SVR can predict flow stress data and reduce the interpolation interval to enhance the simulation accuracy.

\subsection{Construction of three-dimensional (3D) flow stress map}

Ji et al., Zhu et al., and Sabokpa et al. just predicted stress data at some unknown temperatures, certain strain rates and strains $^{27,31,40}$. Quan et al. just developed inconsecutive 3D 


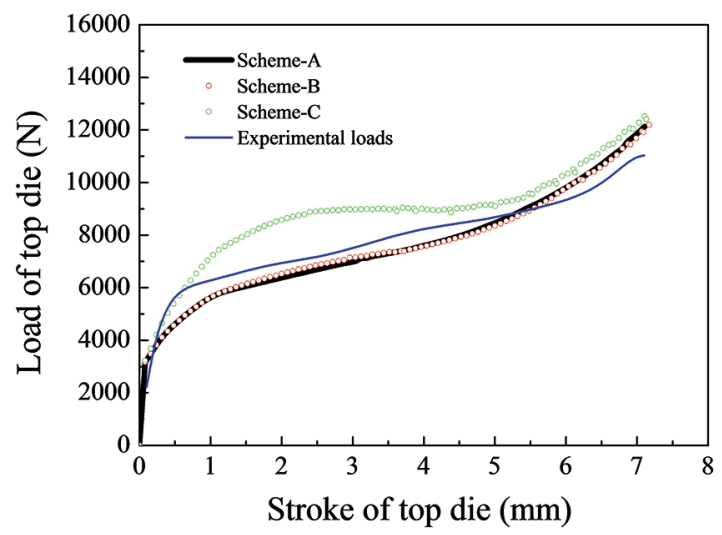

Figure 10: The relationship between the stroke and the loads of top die for these three schemes and experimental values.

map of stress data corresponding to temperature and strain under constant strain rates ${ }^{8}$. In this work, the stress data at temperatures of $1098 \mathrm{~K}, 1148 \mathrm{~K}, 1198 \mathrm{~K}, 1248 \mathrm{~K}$, and $1298 \mathrm{~K}$ under strain rates of $0.01 \mathrm{~s}^{-1}, 0.1 \mathrm{~s}^{-1}, 1 \mathrm{~s}^{-1}$ and 10 $\mathrm{s}^{-1}$ were predicted for Ti-6Al-2Zr-1Mo-1V alloy by the well-trained GA-SVR. Based on the existing experimental stress data and densely predicted stress data, a novel 3D continuous relationships among flow stress, temperature, strain, and strain rate were constructed in Matlab, as shown in Figure 11. Compared with the traditional 2D stress-strain curves, the novel 3D maps of stress data are continuous and can show flow stress data at any strain, strain rate and temperature. As shown in Figure 11, the stress data are displayed by different colors. The $\mathrm{X}$-axis, Y-axis and $\mathrm{Z}$-axis coordinates represent temperature, strain rate and strain, respectively. Figure 11b-d are cross sections of Figure $11 \mathrm{a}$ in three orientations. Figure $11 \mathrm{~b}$ shows the flow stress data corresponding to any strain and strain rate at several fixed temperatures. In can be seen that the stress level increases with the increase of strain rate at a fixed strain, which cannot be visually demonstrated in the traditional 2D stress-strain curves. Figure 11c exhibits the stress data corresponding to any strain and temperature at several fixed strain rates, and it shows that the stress level decreases with increasing temperature at a fixed strain. Figure 11d displays the corresponding stress data to any strain rate and temperature at several fixed strains.

The above mentioned analysis indicates that the flow stress data predicted by the GA-SVR outside of experimental conditions exhibit similar discipline with the experimental stress-strain curves, which can enrich flow stress data and reduce interpolation intervals. And the continuously 3D prediction maps can be converted into a database which can exhibit flow stress data at any strain, strain rate, and temperature. GA-SVR has potential to import into FEM software by using programming language, which is possible to achieve high accuracy of simulations and improve the processing maps, ductile fracture criteria, and the related studies without resorting to time-consuming and high-cost experiments.

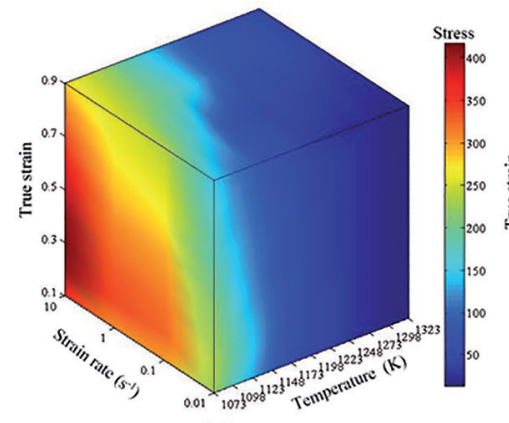

(a)

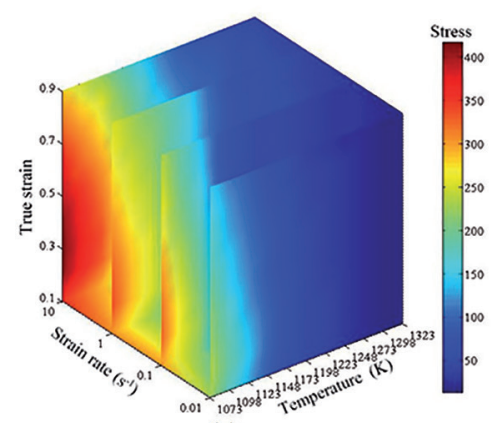

(c)

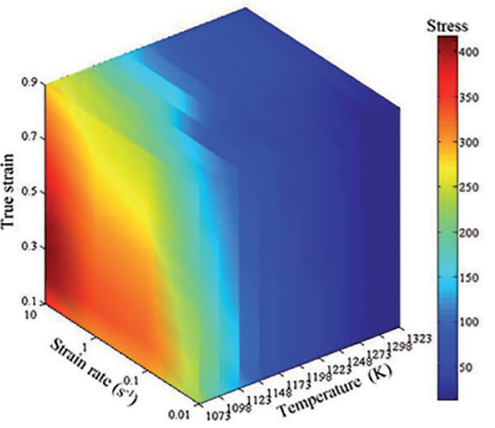

(b)

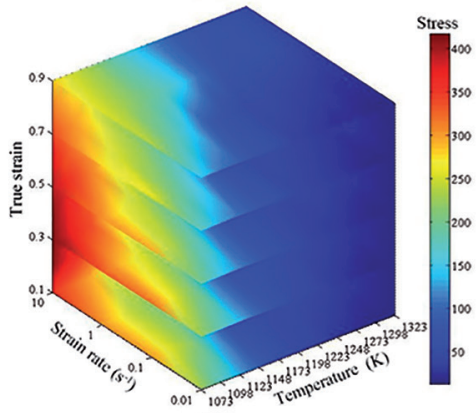

(d)

Figure 11: The (a) three-dimensional stress map and the cross sections at different (b) temperatures, (c) strain rates and (d) strains. 


\section{Conclusions}

The novel prediction model GA-SVR was established to characterize the hot flow behaviors of Ti-6Al-2Zr-1Mo$1 \mathrm{~V}$ alloy according to the experimental stress-strain data. Following conclusions were concluded from the current study:

(1) The complexity, learning ability, and generalization ability of SVR depend on the three parameters $C, \gamma$, and $\zeta$, especially the mutual influence among the three parameters. The SVR with suitable parameters $C, \gamma$, and $\zeta$ will accurately learn the stress-strain curves and appropriately ignore some singular points of stress-strain data to accord with the overall trend of the stress-strain curves.

(2) The average $R$-value \& AARE-value between the training samples and fitted values of the GA-SVR is 0.999963 \& $0.3282 \%$, which show the GA-SVR model can sufficiently and accurately learn the hot flow behaviors which accompany with WH, DRX and DRV. Comparison results show that the study ability of the GA-SVR is as strong as the ANN.

(3) In the comparisons of generation abilities of these models, the GA-SVR has larger $R$-value and lower $A A R E$ value, which indicate that the GA-SVR can accurately predict the highly non-linear flow behaviors of Ti-6Al-2Zr-1Mo-1V alloy. The generation abilities of these models were shown as follows in ascending order: the improved Arrhenius-type constitutive model $<$ ANN $<$ GA-SVR.

(4) Based on the operators of selection, crossover, and mutation, the GA-SVR can self-adaptively and dynamically adjust the processes of selection, crossover, and mutation to realize the optimal selection of the three parameters, which greatly improves the computational efficiency. The modeling efficiencies of these models were shown as follows in ascending order: the improved Arrhenius-type constitutive model $<$ ANN $<$ GA-SVR.

(5) The flow behaviors under different temperature ranges of a material are highly non-linear, therefore, calculating stress data by interpolation method in FEM software is inaccurate. The GA-SVR can predict flow stress data and reduce the interpolation interval to enhance the simulation accuracy without resorting to time-consuming and high-cost experiments. The continuously $3 \mathrm{D}$ relationships among flow stress, temperature, strain, and strain rate were constructed, which can improve the related research fields where stressstrain data play important roles, such as improving the accuracy of finite element simulation result, improving processing maps, characterizing dynamic recrystallization evolution, etc.

\section{Acknowledgements}

This work was supported by National Natural Science Foundation of China (51305469). The corresponding author was also appreciated for Chongqing Higher School Youth-Backbone Teacher Support Program.

\section{References}

1. Wu CB, H Yang, XG Fan, ZC Sun. Dynamic globularization kinetics during hot working of TA15 titanium alloy with colony microstructure. Transactions of Nonferrous Metals Society of China. 2011;21(9):1963-1969. http://dx.doi.org/10.1016/ s1003-6326(11)60957-6.

2. Li L, Ye B, Liu S, Hu S, Li B. Inverse analysis of the stress-strain curve to determine the materials models of work hardening and dynamic recovery. Materials Science and Engineering: A . 2015;636:243-248. http://dx.doi.org/10.1016/j.msea.2015.03.115.

3. Quan GZ, Wang Y, Yu CT, Zhou J. Hot workability characteristics of as-cast titanium alloy Ti-6Al-2Zr-1Mo-1V: A study using processing map. Materials Science and Engineering: A. 2013;564:46-56. http://dx.doi.org/10.1016/j.msea.2012.11.070.

4. Quan GZ, Li GS, Chen T, Wang YX, Zhang YW, Zhou J. Dynamic recrystallization kinetics of $42 \mathrm{CrMo}$ steel during compression at different temperatures and strain rates. Materials Science and Engineering: A. 2011;528(13-14):4643-4651. http://dx.doi. org/10.1016/j.msea.2011.02.090.

5. Quan GZ, Kang BS, Ku TW, Song WJ. Identification for the optimal working parameters of $\mathrm{Al}-\mathrm{Zn}-\mathrm{Mg}-\mathrm{Cu}$ alloy with the processing maps based on DMM. The International Journal of Advanced Manufacturing Technology. 2011;56(9):1069-1078. http://dx.doi.org/10.1007/s00170-011-3241-6.

6. Lin YC, Li QF, Xia YC, Li LT. A phenomenological constitutive model for high temperature flow stress prediction of $\mathrm{Al}-\mathrm{Cu}-\mathrm{Mg}$ alloy. Materials Science and Engineering: A. 2012;534:654-662. http://dx.doi.org/10.1016/j.msea.2011.12.023.

7. Li HY, Hu JD, Wei DD, Wang XF, Li YH. Artificial neural network and constitutive equations to predict the hot deformation behavior of modified 2.25Cr-1Mo steel. Materials \& Design. 2012;42:192-197. http://dx.doi.org/10.1016/j.matdes.2012.05.056.

8. Quan GZ, Lv WQ, Mao YP, Zhang YW, Zhou J. Prediction of flow stress in a wide temperature range involving phase transformation for as-cast $\mathrm{Ti}-6 \mathrm{Al}-2 \mathrm{Zr}-1 \mathrm{Mo}-1 \mathrm{~V}$ alloy by artificial neural network. Materials \& Design. 2013;50:51-61. http://dx.doi.org/10.1016/j.matdes.2013.02.033.

9. Guan Z, Ren M, Zhao P, Ma P, Wang O. Constitutive equations with varying parameters for superplastic flow behavior of Al-ZnMg-Zr alloy. Materials \& Design (1980-2015). 2014;54:906-913. http://dx.doi.org/10.1016/j.matdes.2013.09.014.

10. Fan XG, Yang H, Gao PF. Prediction of constitutive behavior and microstructure evolution in hot deformation of TA15 titanium alloy. Materials \& Design. 2013;51:34-42. http:// dx.doi.org/10.1016/j.matdes.2013.03.103.

11. Voyiadjis GZ, Abed FH. Microstructural based models for bcc and fcc metals with temperature and strain rate dependency. Mechanics of Materials. 2005;37(2-3):355-378. http://dx.doi. org/10.1016/j.mechmat.2004.02.003.

12. Zhang C, Ding J, Dong Y, Zhao G, Gao A, Wang L. Identification of friction coefficients and strain-compensated Arrhenius-type constitutive model by a two-stage inverse analysis technique. International Journal of Mechanical Sciences. 2015;98:195-204. http://dx.doi.org/10.1016/j.ijmecsci.2015.04.015. 
13. Lin YC, Wen DC, Huang YC, Chen XM, Chen XW. A unified physically based constitutive model for describing strain hardening effect and dynamic recovery behavior of a Ni-based superalloy. Journal of Materials Research. 2015;30(24):37843794. http://dx.doi.org/10.1557/jmr.2015.368.

14. Lin YC, Chen XM, Wen DX, Chen MS. A physically-based constitutive model for a typical nickel-based superalloy. Computational Materials Science. 2014;83:282-289. http:// dx.doi.org/10.1016/j.commatsci.2013.11.003.

15. Xiao J, Li DS, Li XQ, Deng TS. Constitutive modeling and microstructure change of Ti-6Al-4V during the hot tensile deformation. Journal of Alloys and Compounds. 2012;541:346352. http://dx.doi.org/10.1016/j.jallcom.2012.07.048.

16. Peng W, Zeng W, Wang Q, Yu H. Comparative study on constitutive relationship of as-cast Ti60 titanium alloy during hot deformation based on Arrhenius-type and artificial neural network models. Materials \& Design. 2013;51:95-104. http://dx.doi.org/10.1016/j.matdes.2013.04.009.

17. Sajadifar SV, Yapici GC. Workability characteristics and mechanical behavior modeling of severely deformed pure titanium at high temperatures. Materials \& Design. 2014;53:749757. http://dx.doi.org/10.1016/j.matdes.2013.07.057.

18. Lin YC, Chen MS, Zhong J. Prediction of $42 \mathrm{CrMo}$ steel flow stress at high temperature and strain rate. Mechanics Research Communications. 2008;35(3):142-150. http:// dx.doi.org/10.1016/j.mechrescom.2007.10.002.

19. Lin YC, Li KK, Li HB, Chen J, Chen XM, Wen DX. New constitutive model for high-temperature deformation behavior of inconel 718 superalloy. Materials \& Design. 2015;74:108118. http://dx.doi.org/10.1016/j.matdes.2015.03.001.

20. Quan GZ, Shi Y, Yu CT, Zhou J. The improved Arrhenius model with variable parameters of flow behavior characterizing for the as-cast AZ80 magnesium alloy. Materials Research. 2013;16(4):785-791. http://dx.doi.org/10.1590/s151614392013005000070 .

21. Khan AS, Kazmi R, Farrokh B, Zupan M. Effect of oxygen content and microstructure on the thermo-mechanical response of three Ti-6Al-4V alloys: Experiments and modeling over a wide range of strain-rates and temperatures. International Journal of Plasticity. 2007;23(7):1105-1125. http://dx.doi. org/10.1016/j.ijplas.2006.10.007.

22. Khan AS, Suh YS, Kazmi R. Quasi-static and dynamic loading responses and constitutive modeling of titanium alloys. International Journal of Plasticity. 2004;20(12):2233-2248. http://dx.doi.org/10.1016/j.ijplas.2003.06.005.

23. Kotkunde N, Deole AD, Gupta AK, Singh SK. Comparative study of constitutive modeling for Ti-6Al-4V alloy at low strain rates and elevated temperatures. Materials \& Design. 2014;55:999-1005. http://dx.doi.org/10.1016/j. matdes.2013.10.089.

24. Akbari Z, Mirzadeh H, Cabrera JM. A simple constitutive model for predicting flow stress of medium carbon microalloyed steel during hot deformation. Materials \& Design. 2015;77:126-131. http://dx.doi.org/10.1016/j. matdes.2015.04.005
25. Liu J, Zeng W, Lai Y, Jia Z. Constitutive model of Ti17 titanium alloy with lamellar-type initial microstructure during hot deformation based on orthogonal analysis. Materials Science and Engineering: A. 2014;597:387-394. http://dx.doi.org/10.1016/j.msea.2013.12.076.

26. Quan GZ, Zhang ZH, Pan J, Xia YF. Modelling the Hot Flow Behaviors of AZ80 Alloy by BP-ANN and the Applications in Accuracy Improvement of Computations. Materials Research. 2015;18(6):1331-1345. http://dx.doi. org/10.1590/1516-1439.040015.

27. Zhu Y, Zeng W, Sun Y, Feng F, Zhou Y. Artificial neural network approach to predict the flow stress in the isothermal compression of as-cast TC21 titanium alloy. Computational Materials Science. 2011;50(5):1785-1790. http://dx.doi. org/10.1016/j.commatsci.2011.01.015.

28. Lou Y, Ke CX, Li L. Accurately Predicting High Temperature Flow Stress of AZ80 Magnesium Alloy with Particle Swarm Optimization-based Support Vector Regression. Applied Mathematics \& Information Sciences. 2013;7(3):1093-1102.

29. Desu RK, Guntuku SC, Aditya B, Gupta AK. Support Vector Regression based Flow Stress Prediction in Austenitic Stainless Steel 304. Procedia Materials Science. 2014;6:368375. http://dx.doi.org/10.1016/j.mspro.2014.07.047.

30. Sun Z, Yang H, Tang Z. Microstructural evolution model of TA15 titanium alloy based on BP neural network method and application in isothermal deformation. Computational Materials Science. 2010;50(2):308-318. http://dx.doi. org/10.1016/j.commatsci.2010.08.020.

31. Ji G, Li F, Li Q, Li H, Li Z. A comparative study on Arrhenius-type constitutive model and artificial neural network model to predict high-temperature deformation behaviour in Aermet100 steel. Materials Science and Engineering: A. 2011;528(13-14):4774-4782. http://dx.doi. org/10.1016/j.msea.2011.03.017.

32. Mandal S, Sivaprasad PV, Venugopal S, Murthy KPN. Artificial neural network modeling to evaluate and predict the deformation behavior of stainless steel type AISI 304L during hot torsion. Applied Soft Computing. 2009;9(1):237244. http://dx.doi.org/10.1016/j.asoc.2008.03.016.

33. Sun Y, Zeng WD, Zhao YQ, Han YF, Ma X. Intelligent method to develop constitutive relationship of Ti-6Al$2 \mathrm{Zr}-1 \mathrm{Mo}-1 \mathrm{~V}$ alloy. Transactions of Nonferrous Metals Society of China. 2012;22(6):1457-1461. http://dx.doi. org/10.1016/s1003-6326(11)61341-1.

34. Quan GZ, Wu DS, Luo GC, Xia YF, Zhou J, Liu Q et al. Dynamic recrystallization kinetics in $\alpha$ phase of as-cast Ti-6Al-2Zr-1Mo-1V alloy during compression at different temperatures and strain rates. Materials Science and Engineering: A. 2014;589:23-33. http://dx.doi.org/10.1016/j. msea.2013.09.069.

35. Sakai T, Belyakov A, Kaibyshev R, Miura H, Jonas JJ. Dynamic and post-dynamic recrystallization under hot, cold and severe plastic deformation conditions. Progress in Materials Science. 2014;60(1):130-207. http://dx.doi. org/10.1016/j.pmatsci.2013.09.002. 
36. Keerthi SS, Lin CJ. Asymptotic behaviors of support vector machines with Gaussian kernel. Neural Computation. 2003;15(7):1667-1689.

37. Sivaraj R, Ravichandran T. An improved clustering based genetic algorithm for solving complex NP problems. Journal of Computer Science. 2011;7(7):1033-1037.

38. Chai RX, Guo C, Yu L. Two flowing stress models for hot deformation of XC45 steel at high temperature. Materials Science and Engineering: A. 2012;534:101-110. http:// dx.doi.org/10.1016/j.msea.2011.11.047.
39. Li LX, Peng DS, Liu JA, Liu ZQ. An experiment study of the lubrication behavior of graphite in hot compression tests of Ti-6Al-4V alloy. Journal of Materials Processing Technology. 2001;112(1):1-5.

40. Sabokpa O, Zarei-Hanzaki A, Abedi HR, Haghdadi N. Artificial neural network modeling to predict the high temperature flow behavior of an AZ81 magnesium alloy. Materials \& Design. 2012;39:390-396. http://dx.doi. org/10.1016/j.matdes.2012.03.002. 